\title{
Aprepitant Sensitizes Acute Myeloid Leukemia Cells to the Cytotoxic Effects of Cytosine Arabinoside in vitro and in vivo
}

This article was published in the following Dove Press journal: Drug Design, Development and Therapy

\author{
Hongzhang $\mathrm{Wu}^{1, *}$ \\ Xurui Cheng ${ }^{1} *$ \\ Feiyan Huang ${ }^{2}$ \\ Gang Shao $\mathbb{D}^{3}$ \\ Yueming Meng' \\ Lingfei Wang ${ }^{3}$ \\ Tao Wang' \\ Xiaoyuan Jia' \\ Tianxin Yang ${ }^{4}$ \\ Xi Wang $\left.{ }^{3}\right)^{3}$ \\ Caiyun Fu $\mathbb{D D}^{1}$
}

'Zhejiang Provincial Key Laboratory of Silkworm Bioreactor and Biomedicine, College of Life Sciences and Medicine, Zhejiang Sci-Tech University, Hangzhou 310018 , People's Republic of China; ${ }^{2}$ Clinical Laboratory, The First Affiliated Hospital of Zhejiang Chinese Medical University, Hangzhou 310006, People's Republic of China; ${ }^{3}$ Department of Oncology, The 903rd Hospital of PLA, Hangzhou 310013, People's Republic of China; ${ }^{4}$ Department of Hematology, Zhejiang Province People's Hospital, Hangzhou 310014, People's Republic of China

*These authors contributed equally to this work
Correspondence: Caiyun Fu Email fucy03@zstu.edu.cn
Purpose: Acute myeloid leukemia (AML) is a complex malignancy characterized by the clonal expansion of immature myeloid precursors. The standard treatment for newly diagnosed AML is chemotherapy consisting of cytosine arabinoside (Ara-C) and anthracyclines with disappointing clinical outcomes and severe adverse effects, such as symptomatic bradycardia, neurotoxicity. Thus, it is promising to treat AML through combination drug therapy to reduce the adverse effects of chemotherapeutics. In our recent published PNAS paper, we reported that NK-1R antagonists, both Aprepitant and SR140333, induce apoptosis of myeloid leukemia cells by inducing oxidative stress through mitochondrial calcium overload. We, therefore, tested the hypothesis of the combination Ara-C with NK-1R antagonist could enhance the efficacy of Ara-C.

Methods: MTT assay was employed to detect the cell proliferation. Flow cytometry was applied to detect the cell cycle and necrosis. PI uptake and LDH release assay were used to detect the disintegration of the plasma membrane. Xenograft model was constructed to explore the effect of combination Ara-C with Aprepitant in vivo.

Results: Our results showed that Aprepitant sensitizes HL60 cells to the cytotoxic effects of Ara-C more than 5-fold by enhancing G0/G1 cell cycle arrest and necrosis in vitro. Furthermore, Nec-1, a specific inhibitor of necroptosis, could recover the cell proliferative viability significantly. Attractively, once every 2-days regimen of Ara-C (5 mg/kg) and Aprepitant $(10 \mathrm{mg} / \mathrm{kg})$ via in situ injection dramatically reduced the tumor volume from $2175.0 \pm 341.9 \mathrm{~mm}^{3}$ in the vehicle group to $828.4 \pm 232.4 \mathrm{~mm}^{3}$ in the combination group without obvious toxicity in human myeloid leukemia xenograft mice.

Conclusion: Taken together, reduced dose of Ara-C combination with moderate Aprepitant provides more effective therapeutical methods for AML treatment in vitro and in vivo with the elimination of the toxicity of Ara-C, which may pay new avenue for the usage of the routine chemotherapy drug Ara-C with low dose to enhance efficacy and reduce toxicity in clinical practice.

Keywords: neurokinin receptor antagonist, cytosine arabinoside, combined chemotherapy, acute myeloid leukemia

\section{Introduction}

Acute myeloid leukemia (AML) is a complex malignancy with significantly genetic mutation and deleterious epigenetic differentiation characterized by the clonal expansion of immature myeloid precursors. ${ }^{1-3}$ The 5-year survival rates for AML are 5-15\% in patients aged 60 years and older, as well as $35-40 \%$ for younger patients. ${ }^{4,5}$ Until now, the main treatment for AML is allogenic hematopoietic cell transplantation and 
chemotherapy consisting of cytosine arabinoside (Ara-C) and anthracyclines. ${ }^{4}$ However, the chemotherapy strategy of anthracyclines and Ara-C remains disappointing clinical outcomes and severe adverse effects, such as symptomatic bradycardia, neurotoxicity, etc. ${ }^{6,7}$ Thus, it is promising to treat AML through combination drug therapy to reduce the adverse effects of chemotherapeutics. ${ }^{8}$

Neurokinin 1 receptor (NK-1R) is a member of G protein-coupled receptors. ${ }^{9} \mathrm{NK}-1 \mathrm{R}$ participates in many biological processes and contributes to multiple disease processes, such as pain, cardiovascular system, affective and addictive disorders, and cancers. ${ }^{10-13} \mathrm{NK}-1 \mathrm{R}$ is overexpressed in many cancers, such as colon cancer, ${ }^{14}$ breast cancer, ${ }^{15}$ osteosarcoma, ${ }^{16}$ glioblastoma, ${ }^{17}$ and gallbladder cancer, ${ }^{18}$ etc. NK-1R antagonists are promising for the treatment of cancers with overexpression NK-1R, ${ }^{19}$ in which Aprepitant is the first antagonist approved by the FDA to treat chemotherapy-induced nausea and vomiting. ${ }^{20,21}$ For myeloid leukemia treatment, our recent results showed that NK-1R antagonists, both Aprepitant and SR140333, induce apoptosis of myeloid leukemia cells by inducing oxidative stress through mitochondrial calcium overload. ${ }^{22}$

Based on the fact chemotherapeutics Ara-C with severe adverse effects, in the present study, we investigated the combination efficacy of Ara-C with Aprepitant in vitro and in vivo in order to reduce the dosage of Ara-C in AML treatment.

\section{Materials and Methods Cell Line and Drugs}

Human acute myeloid leukemia cell line HL60 cells were obtained from the Chinese Academy of Medical Sciences. Ara-C (Cat\# C8040) was purchased from Solarbio and dissolved in PBS. Aprepitant (Cat\# YZ-1041904) was purchased from Solarbio and dissolved in DMSO (Applichem, Cat\# A3672) as the stock solution, while the content of DMSO in working solution is less than $0.1 \%$. Z-VAD-FMK (Cat\# HY-16658) was purchased from MCE. Necrostatin-1 (Cat\# T1847) was purchased from TargetMol. Cells were cultured in RPMI medium 1640 (Gibco, Cat\# 31800-022).

\section{Cell Viability Assay}

Thiazolyl blue tetrazolium Bromide (MTT) (Solarbio, Cat\# M8180) was used for the assessment of cell viability. HL60 cells during the logarithmic growth phase were inoculated into 96 -well plates at $1 \times 10^{4}$ per well. HL60 cells were then treated with different concentrations of Ara-C, Aprepitant or the combination of both drugs at $37^{\circ} \mathrm{C}$ for 24 hours. At the end of this period, $5 \mathrm{mg} / \mathrm{mL}$ MTT was added to the wells and incubated at $37^{\circ} \mathrm{C}$ for 4 hours. Purple formazan crystals formed in viable cells were solubilized in DMSO. Absorbance was measured at $490 \mathrm{~nm}$ by a Microplate reader (Varioskan Flash, Thermo).

\section{Flow Cytometry}

Cell cycle analysis using Cell Cycle Detection Kit (KeyGEN, Cat\# KGA512) was performed with Ara-C, Aprepitant or the combination of both drugs at $37^{\circ} \mathrm{C}$ for 24 hours. Then, HL60 cells were collected and were washed with PBS two times before fixing with methanol at $-20^{\circ} \mathrm{C}$ overnight. Cells were stained with the mixed solution of PI and RNase A and then incubated at room temperature for $30 \mathrm{~min}$ prior to flow cytometry analysis (BD AccuriтM C6). NovoExpress (aceabio, USA) software was used for analysis results.

Assessment of cell death was detected by Annexin V-FITC/PI double staining Apoptosis Detection Kit (KeyGEN, Cat\# KGA108). HL60 cells were treated with Ara-C, Aprepitant or the combination of both drugs for 24 hours. Cells were harvested and washed with PBS two times, and then resuspended in $500 \mu \mathrm{L}$ of binding buffer containing $5 \mu \mathrm{L}$ Annexin V-FITC and $5 \mu \mathrm{L}$ propidium iodide (PI) for $15 \mathrm{~min}$ at room temperature, followed by Flow Cytometry (BD Accuri ${ }^{\mathrm{TM}}$ C6). NovoExpress (aceabio, USA) software was used for analysis results.

\section{PI Uptake Assay}

HL60 cells during logarithmic growth phase were inoculated into 96 -well plates at $5 \times 10^{4}$ per well, and then treated with Ara-C, Aprepitant or the combination of both drugs for 24 and 48 hours. At the indicated time point, $10 \mathrm{mg} / \mathrm{mL}$ PI (Solarbio, Cat\# P8080) was added to each well and incubated at $37^{\circ} \mathrm{C}$ for $30 \mathrm{~min}$. The photos were recorded using an inverted fluorescence microscope (Axio Vert A1, ZEISS) to count and calculate the proportion of cells absorbing PI.

\section{LDH-Cytotoxicity Assay}

To examine the release of LDH from HL60 cells in supernatant, we used the LDH-cytotoxicity assay kit (Beyotime, Cat\# C0017) according to the manufacturer's protocol as our recent report. ${ }^{8}$ In brief, the supernatant in each group was collected and centrifuged at $400 \mathrm{~g}$ for $5 \mathrm{~min}$, and the amount of LDH released into the medium was measured by the LDH-cytotoxicity assay kit (Beyotime, C0017). The absorbance was measured at $490 \mathrm{~nm}$ by a Microplate reader (Varioskan Flash, Thermo). 


\section{Tumor Xenograft Model}

Animal experiment was conducted strictly in accordance with the Animal Welfare Act approved by the Ethics Committee of Animal Experiments at Zhejiang Sci-Tech University. For the subcutaneous xenograft model, HL60 cells $\left(1 \times 10^{7}\right.$ cells/mouse) were injected subcutaneously into mice. When the tumor volume reached $100 \sim 150 \mathrm{~mm}^{3}$, the mice were randomized into four groups as follows: Vehicle group (PBS and Corn Oil), $5 \mathrm{mg} / \mathrm{kg}$ Ara-C group (dissolved in PBS), $10 \mathrm{mg} / \mathrm{kg}$ Aprepitant group (dissolved in Corn Oil), $5 \mathrm{mg} / \mathrm{kg}$ Ara-C $+10 \mathrm{mg} / \mathrm{kg}$ Aprepitant. The mice in each group were weighed and treated via in situ injection once every 2 days. Tumor volumes were measured and evaluated according to the following formula: tumor volume $\left(\mathrm{mm}^{3}\right)=$ $\left(\right.$ length $\times$ width $\left.^{2}\right) / 2$. When the tumor volume exceeds $2000 \mathrm{~mm}^{3}$, the mice were euthanized and tumor tissues were isolated. Whole blood was collected and analyzed for white blood cells (WBC), red blood cells (RBC), hemoglobin (HGB) and platelets (PLT) by hematology analyzer (BC5390CRP, Mindary).

\section{Statistical Tests}

The significance of the difference of the means was tested by the Student's $t$ test using SPSS software (version 19.0). All statistical data are presented as the mean \pm standard error of the mean (SEM). Differences were considered significant when $P<0.05$.

\section{Result}

\section{Aprepitant Sensitizes HL60 Cells to the Cytotoxic Effects of Ara-C in vitro}

Using human acute myeloid leukemia cell line HL60 cells, our results showed that both Ara-C and Aprepitant inhibited the proliferation of HL60 cells in a dose-dependent manner (Figure 1A). The intensity of proliferative viability was 89.17 $\pm 3.92 \%, 75.84 \pm 2.93 \%, 74.16 \pm 1.86 \%, 69.16 \pm 2.38 \%$ and $62.18 \pm 2.63 \%$ after treatment with Ara-C for 24 hours at 0.4 $\mu \mathrm{M}, 0.8 \mu \mathrm{M}, 1.2 \mu \mathrm{M}, 1.6 \mu \mathrm{M}$ and $2.0 \mu \mathrm{M}$, respectively (Figure 1A). Consistent with our recent reports, ${ }^{22}$ Aprepitant also inhibited the proliferation of HL60 cells in a dose-dependent manner with the proliferative viability of $85.98 \pm 2.53 \%, 71.07 \pm 2.36 \%, 52.37 \pm 0.95 \%, 50.47 \pm$ $0.89 \%$ and $23.07 \pm 1.58 \%$ for 24 hours at $5 \mu \mathrm{M}, 10 \mu \mathrm{M}, 15$ $\mu \mathrm{M}, 20 \mu \mathrm{M}$ and $30 \mu \mathrm{M}$, respectively (Figure 1A). However, the proliferative viability of combination of both Ara-C at 0.4 $\mu \mathrm{M}$ and Aprepitant at $10 \mu \mathrm{M}$ was $41.83 \pm 3.96 \%$, while the proliferative viability of combination of both Ara-C at 0.8 $\mu \mathrm{M}$ and Aprepitant at $10 \mu \mathrm{M}$ was $34.90 \pm 1.87 \%$, indicating that the combination efficacy is much more potent than each single dose (Figure 1B). Aprepitant sensitized HL60 cells to the cytotoxic effects of Ara-C with 5.37 fold $(0.4 \mu \mathrm{M}$ Ara-C) and 2.69 fold $(0.8 \mu \mathrm{M}$ Ara-C) (Figure $1 \mathrm{~A}$ and $\mathrm{B})$. The representative pictures corresponding to each group are shown in Figure 1C.

\section{Aprepitant Enhances G0/GI Cell Cycle Arrest of HL60 Cells Treated by Ara-C}

In order to clarify the molecular mechanisms involved in the chemosensitivity of Ara-C combination with Aprepitant, we first detected cell cycle distribution using flow cytometry. Our results showed that Ara-C $(0.8 \mu \mathrm{M})$ and Aprepitant (10 $\mu \mathrm{M})$ induced cell cycle arrest significantly in $\mathrm{G} 0 / \mathrm{G} 1$ phase with the potential of $69.63 \pm 1.08 \%$ and $54.96 \pm 2.06 \%$, respectively, compared with the G0/G1 phase distribution of $37.14 \pm 2.02 \%$ in the control group (Figure 2A and B). However, the combination of Ara-C and Aprepitant markedly enhanced cell cycle arrest with the G0/G1 phase distribution of $76.02 \pm 1.51 \%$ comparing with Ara-C group or Aprepitant group alone (Figure 2A and B).

\section{Aprepitant Enhances Necrosis of HL60 Cells Treated by Ara-C}

In order to further investigate the molecular mechanisms involved in the chemosensitivity of Ara-C combination with Aprepitant, we next detected apoptosis and necrosis by both Annexin V-FITC/PI double-labeled cytometry. ${ }^{8}$ Our results showed that HL60 cells with PI (propidium iodide) positive staining (Q1 area) were increased significantly after combined treatment with Ara-C and Aprepitant (Figure $3 \mathrm{~A}$ and $\mathrm{B}$ ), indicating that Aprepitant enhances the necrosis of HL60 cells treated by Ara-C. However, Aprepitant could not enhance the apoptosis (Q2 and Q4 area) significantly after Ara-C treated (Figure $3 \mathrm{~A}$ and $\mathrm{B}$ ). PI uptake and LDH release are two distinct assays to detect disintegration of the plasma membrane to monitor necrosis in response to cytotoxic insults. ${ }^{8,23}$ Furthermore, the results of PI uptake assay using fluorescence microscope showed that treatment of HL60 cells with both Ara-C and Aprepitant induced a significant increase of PI uptake cells from 11.06 $\pm 1.90 \%$ (Ara-C alone) to $27.45 \pm 0.88 \%$ (Ara-C combined with Aprepitant) for 24 hours, and from $35.59 \pm 2.57 \%$ (Ara$\mathrm{C}$ alone) to $51.79 \pm 3.03 \%$ (Ara-C combined with Aprepitant) for 48 hours (Figure $3 \mathrm{C}$ and D). Consistently, the results of LDH release showed that the treatment of HL60 
A

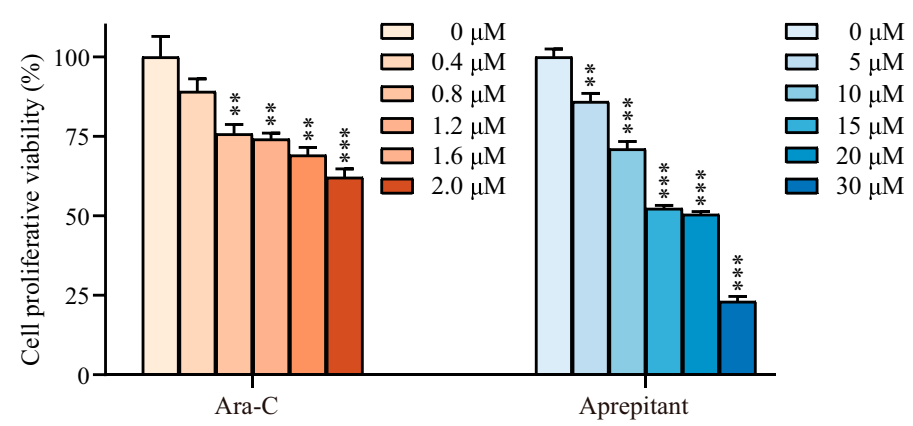

C

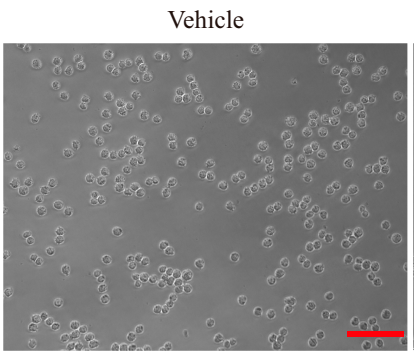

Ara-C $(0.4 \mu \mathrm{M})+$ Aprepitant

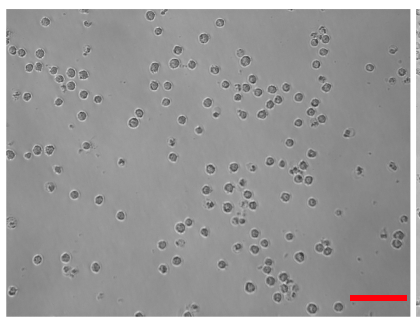

B

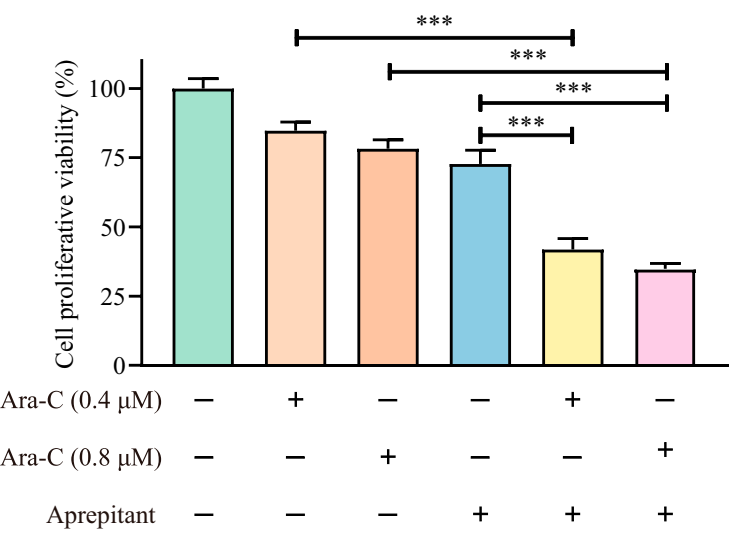

Figure I Aprepitant sensitizes HL60 cells to the cytotoxic effects of Ara-C in vitro. (A) The proliferative viability of HL60 cells after treatment with Ara-C or Aprepitant at the indicated doses for 24 hours. The cell viability was calculated as the percentage of live cells in the drug treatment group relative to the $0 \mu M$ group by MTT assay. Values represent mean $\pm \operatorname{SEM}(n=6)$. $* * P<0.01$, $* * * P<0.00$ I, compared with the control group. $(B)$ Cell proliferative viability was measured by $M T T$ assay after treatment with 0.4 $\mu \mathrm{M}$ Ara-C, $0.8 \mu \mathrm{M}$ Ara-C, $10 \mu \mathrm{M}$ Aprepitant, and the combination of two drugs for 24 hours. Values represent mean \pm SEM ( $\mathrm{n}=6$ ), $* * * P<0.00 \mathrm{I}$, compared with each indicated group. (C) Microscopic observation of the effect of Ara-C combined with Aprepitant on HL60 cells for 24 hours, Scale bar, $50 \mu \mathrm{m}$.

cells with both Ara-C and Aprepitant induced a significant increase of LDH release in HL60 cells (Figure 3E). Taken together, Aprepitant has a powerful effect to enhance the necrosis induced by Ara-C in HL60 cells. Necrostatin-1 (Nec-1) is a specific inhibitor of necroptosis. ${ }^{24}$ After treatment with Nec-1, the necrosis induced by combination Ara-C and Aprepitant was recovered significantly (Figure $3 \mathrm{~F}$ ). Z-VAD-FMK, a specific caspase inhibitor, is a key compound for studies on apoptosis. ${ }^{25}$ Both Ara-C and Aprepitant could induce moderate late apoptosis (Figure 3A and $\mathrm{B}$ ), so we also used Z-VAD-FMK alone or combination with Nec-1 to detect whether they have the ability to rescue cell death of HL60 cells treated by Ara-C and Aprepitant. In
Figure 3F, we can see that Z-VAD-FMK alone had the ability to rescue cell death moderately, while combination Z-VADFMK and Nec-1 could recover the cell proliferative viability significantly from $28.97 \pm 2.41 \%$ before treatment and 57.72 $\pm 2.02 \%$ after treatment with these two distinct inhibitors.

\section{Aprepitant Enhances the Growth-Inhibiting Activity of Human Myeloid Leukemia Xenograft Treated by Ara-C}

The in vivo effect of combination Ara-C with Aprepitant on human myeloid leukemia cell growth was examined in the 
A
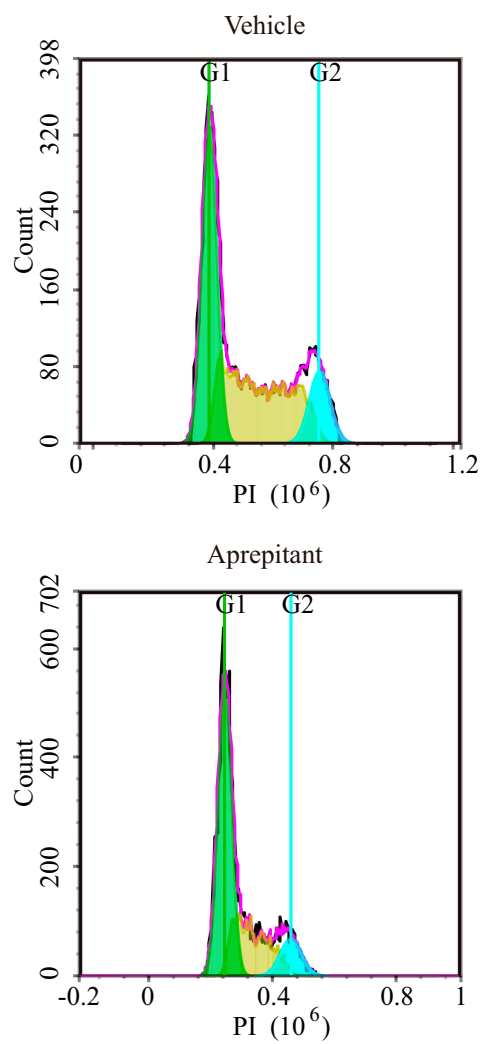

B

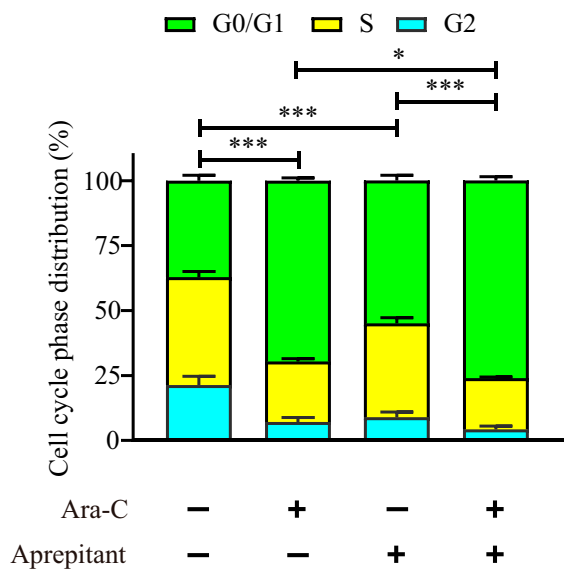

Figure 2 Aprepitant enhances G0/GI cell cycle arrest of HL60 cells treated by Ara-C. (A) Representative images of cell cycle distribution detected by flow cytometry after treatment with $0.8 \mu \mathrm{M}$ Ara-C, $10 \mu \mathrm{M}$ Aprepitant, and combined Ara-C with Aprepitant for 24 hours. (B) The statistical results of cell cycle distribution in HL60 cells. Values represent mean \pm SEM $(n=4) . * P<0.05$, $* * * P<0.001$, compared with each indicated group.

HL60 xenograft mouse model. Once every 2-days regimen of Ara-C (5 mg/kg) and Aprepitant $(10 \mathrm{mg} / \mathrm{kg})$ via in situ injection dramatically reduced the tumor volume from $2175.0 \pm 341.9 \mathrm{~mm}^{3}$ in the vehicle group to $828.4 \pm$ $232.4 \mathrm{~mm}^{3}$ in the combination group at day 10 (Figure 4A and $\mathrm{B})$. However, Ara-C (5 mg/kg) alone could not reduce the tumor volume significantly, just from $2175.0 \pm$ $341.9 \mathrm{~mm}^{3}$ in the vehicle group to $1869.0 \pm 153.5 \mathrm{~mm}^{3}$ in the Ara-C group (Figure 4A and B). Consistently, the tumor weight in the combination group $(0.34 \pm 0.10 \mathrm{~g})$ was reduced significantly compared with Ara-C group $(1.05 \pm 0.11 \mathrm{~g})$ at day 10 after mice euthanasia (Figure 4C).

\section{Assessment of Safety and Toxicity of Combination Ara-C and Aprepitant in Mice}

For the preliminary safety investigation of combination Ara-C and Aprepitant in vivo, we observed the appearance, body weight and fecal and urinary excretion of the mice, and no toxicity was found (Figure 5A and B). After mice euthanasia, organ indexes were calculated by body weight and organ weight to further assess the toxicity of the drugs. There was no significant difference among control group, Ara-C group, Aprepitant group and the combination group in liver index, spleen index, kidney index, heart index, lung index and brain index (Figure 5C). We also conducted a routine blood examination. No significant differences in white blood cells, red blood cells, hemoglobin concentration or platelet quantity were observed among these groups (Figure 5D). Taken together, our present results indicate that the combination of Ara-C and Aprepitant to treat AML is safe in vivo. Moreover, reduced-dose of Ara-C combination with moderate Aprepitant provides a more effective therapeutical method for AML treatment in vitro and in vivo with eliminating the toxicity of Ara-C.

\section{Discussion}

The standard treatment for newly diagnosed AML is 3 days of an anthracycline and 7 days of Ara-C (100-200 mg/m ${ }^{2}$ 
A

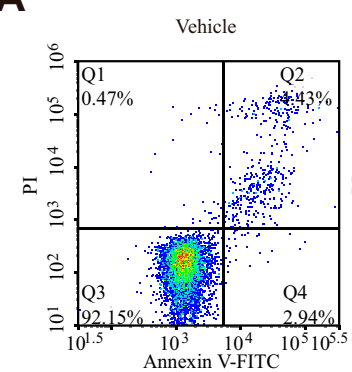

B

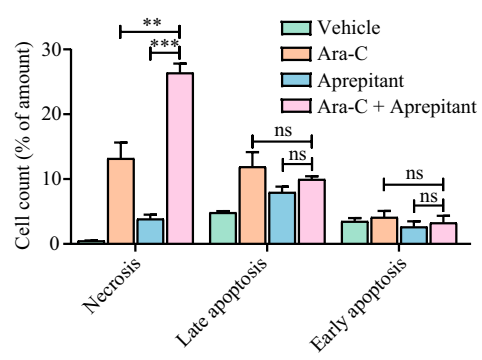

C

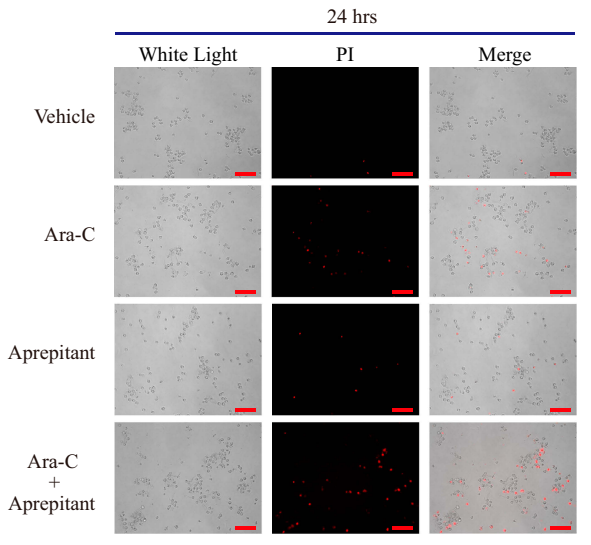

E

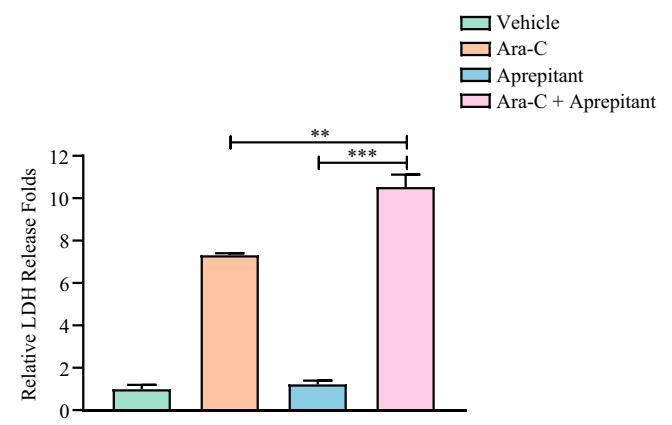

D

D

F

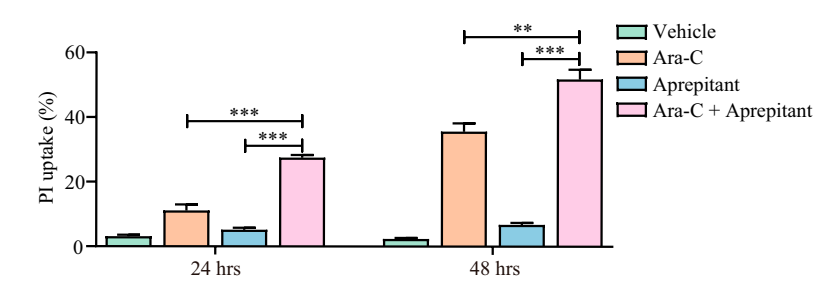

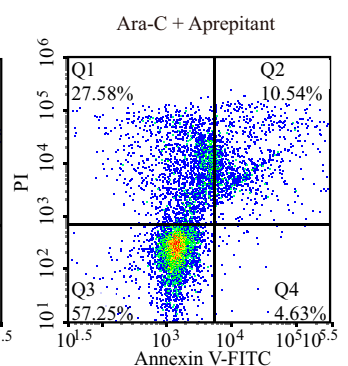
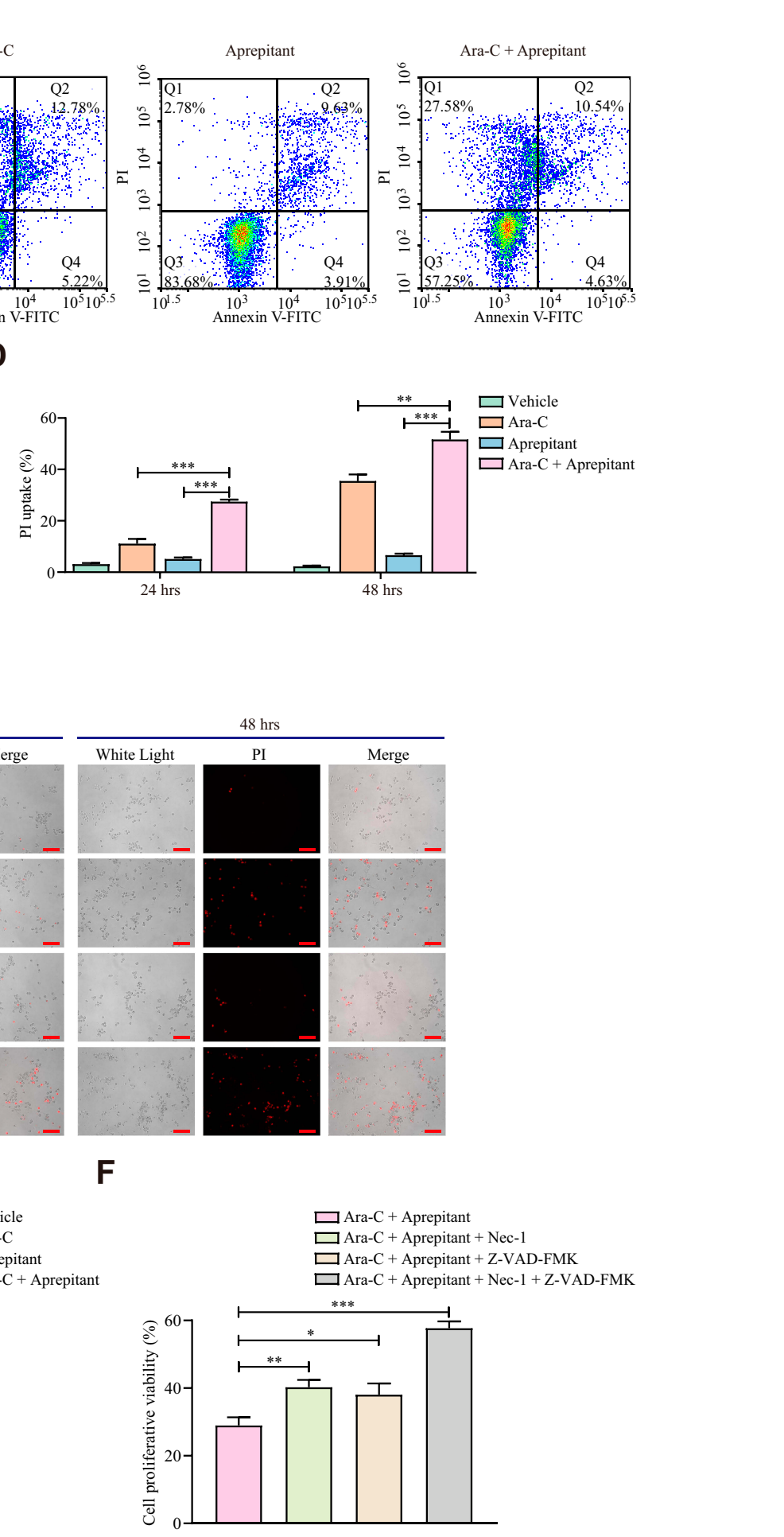

Figure 3 Aprepitant enhances necrosis of HL60 cells treated by Ara-C. (A) Representative images of apoptosis or necrosis for HL60 cells were measured by flow cytometry after treatment with $0.8 \mu \mathrm{M}$ Ara-C, $10 \mu \mathrm{M}$ Aprepitant, and the combination of two drugs for 24 hours. (B) The statistical results of cell death including necrosis (QI area), late apoptosis (Q2 area) and early apoptosis (Q4 area) detected by Annexin- $V$ plus $\mathrm{Pl}$ analysis. Values represent mean $\pm \mathrm{SEM}\left(\mathrm{n}=3\right.$ ). $\mathrm{ns}$ : not significant; ${ }^{* * P}<0.0 \mathrm{I}$, $* * * P<0.00$ I, compared with each indicated group. (C) Microscopic recording of the cell numbers of PI uptake by PI staining after treatment with $0.8 \mu \mathrm{M}$ Ara-C, $10 \mu \mathrm{M}$ Aprepitant, and the combination of two drugs for 24 hours and 48 hours. Scale bar, $50 \mu \mathrm{m}$. (D) The percentage of PI uptake cells was indicated by the cell numbers of PI uptake/the total numbers. Values represent mean $\pm \operatorname{SEM}(n=6)$. $* * P<0.01$, $* * * P<0.00 \mathrm{I}$, compared with the indicated group. (E) The LDH release of HL60 cells was measured by $\mathrm{LDH}$-Cytotoxicity Assay after treatment with $0.8 \mu \mathrm{M}$ Ara-C, I $\mu \mathrm{M}$ Aprepitant, and the combination of two drugs for 24 hours. The relative LDH release folds were indicated by the value of drug treatment group/the value of Vehicle group. Values represent mean $\pm \operatorname{SEM}(n=3)$. $* * P<0.0 \mathrm{I}$, $* * * P<0.00 \mathrm{I}$, compared with the indicated group. (F) The HL60 cells were pretreated with Nec-I $(20 \mu \mathrm{M})$, Z-VAD-FMK $(50 \mu \mathrm{M})$ or Nec-I and Z-VAD-FMK for I hour. Cell viability was measured by MTT assay after treatment with $0.8 \mu \mathrm{M}$ Ara-C and $10 \mu \mathrm{M}$ Aprepitant for 24 hours. Values represent mean $\pm S E M(n=6), * P<0.05$, $* * P<0.01$, $* * * P<0.001$, compared with the indicated group. 
A

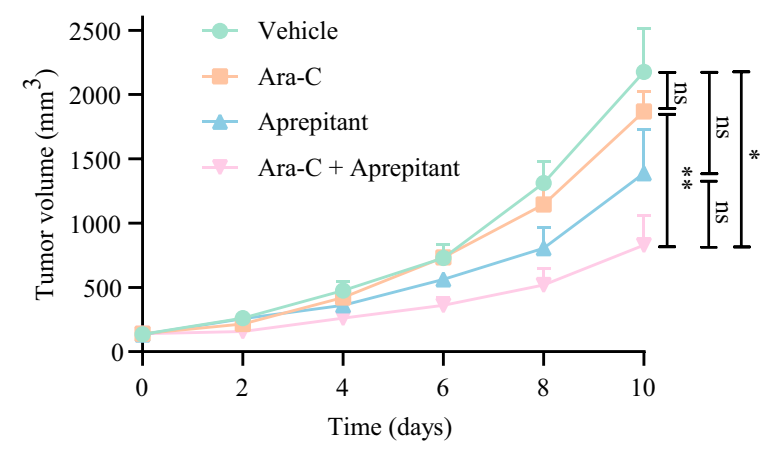

C

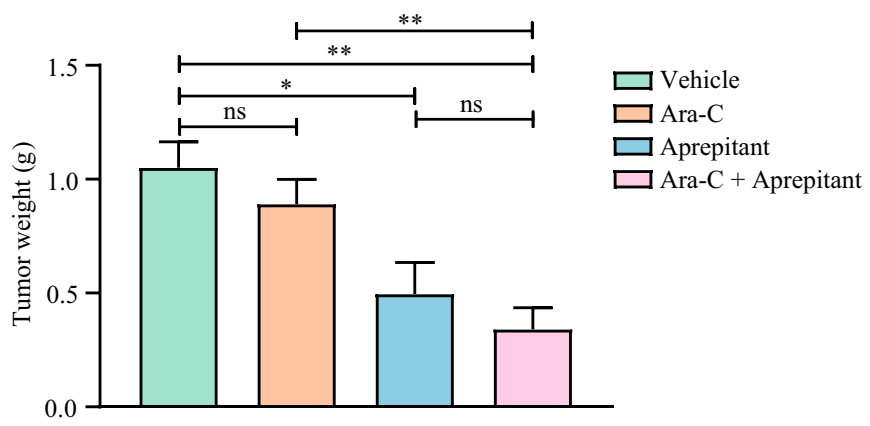

B

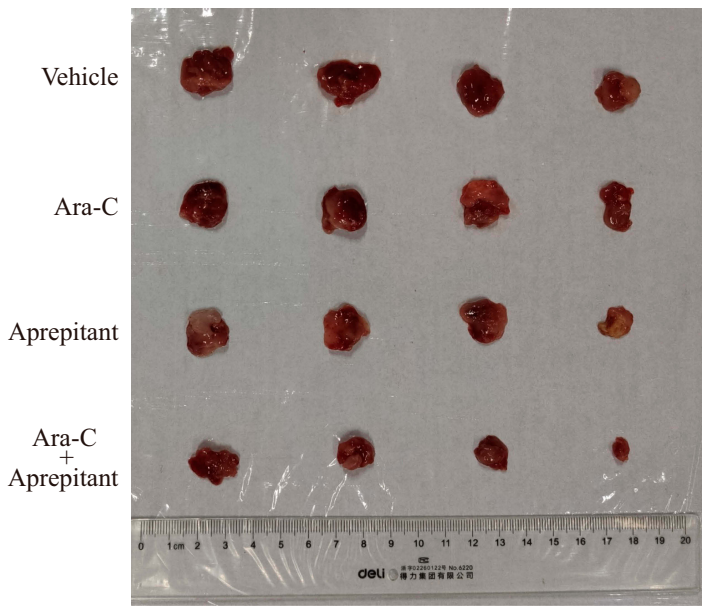

Figure 4 Aprepitant enhances the growth-inhibiting activity of human myeloid leukemia xenograft treated by Ara-C. (A) The tumors volumes measured at the indicated time points. Values represent mean \pm SEM $(n=4)$, ns: not significant; $* P<0.05$, $* * P<0.0$ I, compared with the indicated group. (B) Image of tumors excised from all mice on day 10 ( $n=4$ mice for each group). (C) Each tumor weight excised from all mice on day 10 was calculated. Values represent mean \pm SEM ( $n=4)$, ns: not significant; $* P<0.05, * * P<0.01$, compared with the indicated group.

continuous IV) for more than 30 years. ${ }^{26}$ High-dose Ara-C, for example, $2 \mathrm{~g} / \mathrm{m}^{2}$ every 12 hours on days $1-6^{27}$ or $3 \mathrm{~g} / \mathrm{m}^{2}$ every 12 hours on days $1,3,5$ and $7,{ }^{28}$ shows no higher complete remission $(\mathrm{CR})$ rate and no overall survival differences, but more toxicity in induction with more leukopenia, thrombocytopenia, nausea, vomiting and eye toxicity, compared with standard-dose Ara-C for AML treatment. ${ }^{27,28}$ Thus, it is urgent to develop new combination treatment with reduced-dose of Ara-C to achieve less toxicity as well as the equivalent potential for AML treatment.

$\mathrm{SP} / \mathrm{NK}-1 \mathrm{R}$ system is related to the occurrence and development of many kinds of diseases and NK-1R antagonists are promising for the treatment of diseases. $^{29,30}$ Our recent study showed that NK-1R antagonists have the ability to induce endoplasmic reticulum (ER) stress and then increase mitochondrial ROS to further induce DNA damage response, which mediates the apoptosis of myeloid leukemia cells. ${ }^{22}$ Perturbation of ER homeostasis results in unfolded protein response and targeting ER stress is attractive for disease treatment. ${ }^{31-34} \mathrm{We}$ also assessed the safety of NK-1R antagonists, Aprepitant and SR140333, in AML treatment, and these antagonists showed no obvious toxicity to human normal CD $34^{+}$ hematopoietic cells and human red blood cells. ${ }^{22}$ Thus, in our present study, we detected whether NK-1R antagonist Aprepitant could cooperate with Ara-C to reduce the dosage of Ara-C in order to decrease the toxicity of Ara-C in the clinic. Surprisingly, low-dose of Ara-C combination with Aprepitant is much more potent than Ara-C alone (more than 5 fold in vitro and more than 3 fold in vivo) to mediate the death of HL60 cells via the enhancement of G0/G1 cell cycle arrest and necrosis. Our results thus provide the preclinical evidence to support the combination therapy of Ara-C and Aprepitant, as potential antiAML combination drugs in order to enhance efficacy and reduce toxicity of Ara-C. 
A

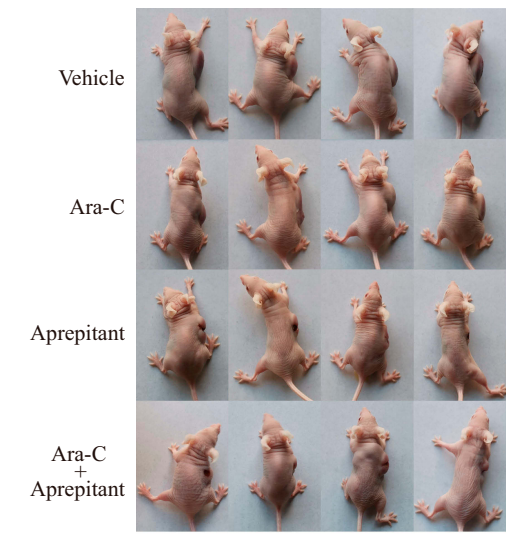

C

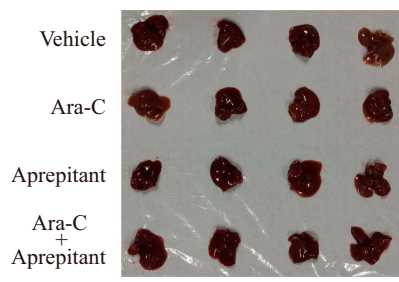

B

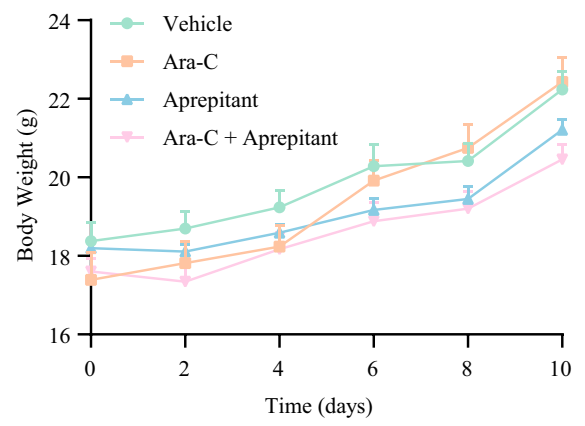

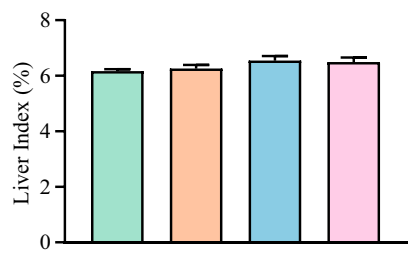
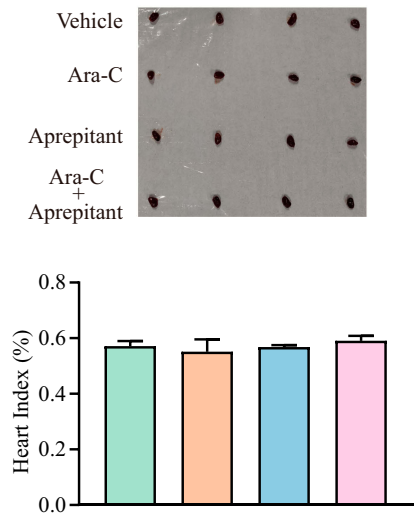
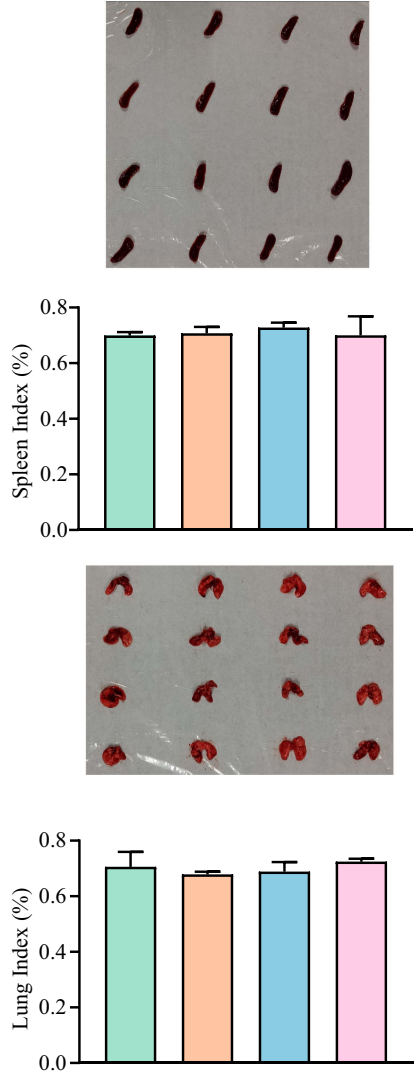
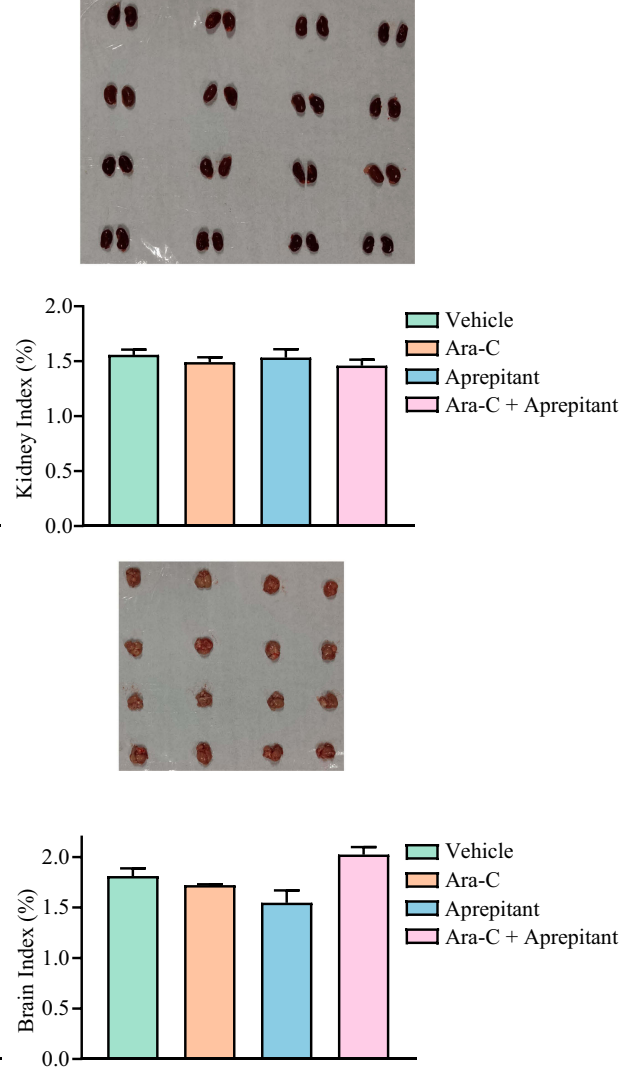

D

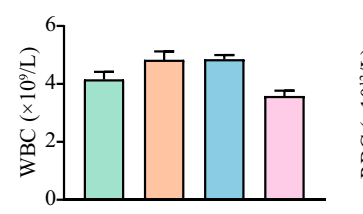

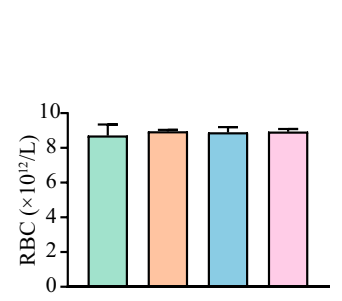
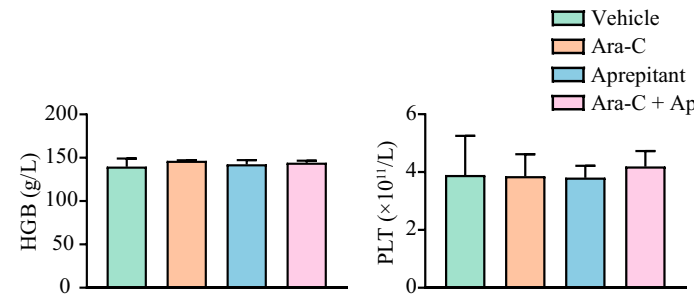

Figure 5 Assessment of safety and toxicity of combination Ara-C and Aprepitant in mice. (A) Images of all mice in each group were photographed before euthanasia at day 10. (B) The weight of mice was measured at the indicated time points. There was no significant difference among each group $(n=4)$. $(C)$ The organs were photographed and the organ index was calculated as organ weight relative to body weight. Values represent mean \pm SEM $(n=4)$. There was no significant difference among each group. (D) The blood parameters including WBC (white blood cells), RBC (red blood cells), HGB (hemoglobin) and PLT (platelets) were analyzed. Values represent mean \pm SEM ( $\mathrm{n}=4$ ). There was no significant difference among each group. 
Taken together, this study revealed the combination chemotherapy of Ara-C and NK-1R antagonist, which provides a therapeutic option for AML treatment with reduced clinical dosage of Ara-C. We also identified the molecular mechanisms involved in the enhancement efficacy of Ara-C via combination with Aprepitant, the NK-1R antagonist approved by the FDA in the clinic for chemotherapy-induced nausea and vomiting treatment. Our present study will not only extend the clinical applications of NK-1R antagonist Aprepitant but also pay new avenue for the usage of the routine chemotherapy drug Ara-C with low-dose to enhance efficacy and reduce toxicity in clinical practice.

\section{Acknowledgments}

This work was supported by grants from the National Natural Science Foundation of China (No. 81770176), the New Century 151 Talent Project of Zhejiang Province, the 521 Talent Foundation and the Fundamental Research Funds of Zhejiang Sci-Tech University (No. 2019Y001), the Science Technology Department of Zhejiang Province (No. LGC19H080001), Science Foundation of Zhejiang Sci-Tech University (No. 18042291-Y).

\section{Disclosure}

The authors declare no competing financial interests exist.

\section{References}

1. Goldman SL, Hassan C, Khunte M, et al. Epigenetic modifications in acute myeloid leukemia: prognosis, treatment, and heterogeneity. Front Genet. 2019;10:133. doi:10.3389/fgene.2019.00133

2. Kansal R. Acute myeloid leukemia in the era of precision medicine: recent advances in diagnostic classification and risk stratification Cancer Biol Med. 2016;13(1):41-54. doi:10.20892/j.issn.20953941.2016.0001

3. Fernandez C, Santos-Silva MC, Lopez A, et al. Newly diagnosed adult AML and MPAL patients frequently show clonal residual hematopoiesis. Leukemia. 2013;27(11):2149-2156. doi:10.1038/ leu.2013.109

4. Dohner H, Weisdorf DJ, Bloomfield CD. Acute myeloid leukemia. N Engl J Med. 2015;373(12):1136-1152. doi:10.1056/NEJMra1406184

5. Oran B, Weisdorf DJ. Survival for older patients with acute myeloid leukemia: a population-based study. Haematologica. 2012;97 (12):1916-1924. doi:10.3324/haematol.2012.066100

6. Wayangankar SA, Patel BC, Parekh HD, Holter JL, Lazzara R. Highdose cytosine arabinoside-induced symptomatic bradycardia. $J$ Cardiovasc Med (Hagerstown). 2015;16(Suppl 1):S38-S41. doi:10.2459/JCM.0b013e328341d0e5

7. Nurgat ZA, Alzahrani H, Lawrence M, et al. Intracranial hypertension secondary to high dose cytosine arabinoside - a case study. J Infect Chemother. 2017;23(5):319-322. doi:10.1016/j.jiac.2016.11.005

8. Lv Y, Shao G, Zhang Q, et al. The antimicrobial peptide PFR induces necroptosis mediated by ER stress and elevated cytoplasmic calcium and mitochondrial ROS levels: cooperation with Ara-C to act against acute myeloid leukemia. Signal Transduct Target Ther. 2019;4:38. doi:10.1038/s41392-019-0073-6
9. Steinhoff MS, von Mentzer B, Geppetti P, Pothoulakis C, Bunnett NW. Tachykinins and their receptors: contributions to physiological control and the mechanisms of disease. Physiol Rev. 2014;94(1):265-301. doi:10.1152/physrev.00031.2013

10. Fu CY, Yang Q, Wang KR, Kong ZQ, Chen Q, Wang R. Rat/mouse hemokinin-1, a mammalian tachykinin peptide, markedly potentiates the antinociceptive effects of morphine administered at the peripheral and supraspinal level. Behav Brain Res. 2006;170(2):293-301. doi:10.1016/j.bbr.2006.03.007

11. Fu CY, Kong ZQ, Long Y, Chen Q, Wang R. Cardiovascular responses to rat/mouse hemokinin-1, a mammalian tachykinin peptide: systemic study in anesthetized rats. Eur J Pharmacol. 2007;572 (2-3):175-181. doi:10.1016/j.ejphar.2007.06.014

12. Fu CY, Zhao YL, Dong L, Chen Q, Ni JM, Wang R. In vivo characterization of the effects of human hemokinin-1 and human hemokinin-1 (4-11), mammalian tachykinin peptides, on the modulation of pain in mice. Brain Behav Immun. 2008;22(6):850-860. doi:10.1016/j.bbi.2007.12.010

13. Munoz M, Covenas R, Esteban F, Redondo M. The substance P/ NK-1 receptor system: NK-1 receptor antagonists as anti-cancer drugs. $J$ Biosci. 2015;40(2):441-463. doi:10.1007/s12038-0159530-8

14. Garnier A, Vykoukal J, Hubertus J, et al. Targeting the neurokinin-1 receptor inhibits growth of human colon cancer cells. Int J Oncol. 2015;47(1):151-160. doi:10.3892/ijo.2015.3016

15. Zhou Y, Zuo D, Wang M, et al. Effect of truncated neurokinin-1 receptor expression changes on the interaction between human breast cancer and bone marrow-derived mesenchymal stem cells. Genes Cells. 2014;19(9):676-691. doi:10.1111/gtc.12168

16. Munoz M, Berger M, Rosso M, Gonzalez-Ortega A, Carranza A, Covenas R. Antitumor activity of neurokinin-1 receptor antagonists in MG-63 human osteosarcoma xenografts. Int J Oncol. 2014;44 (1):137-146. doi:10.3892/ijo.2013.2164

17. Zhang YX, Li XF, Yuan GQ, et al. beta-Arrestin 1 has an essential role in neurokinin-1 receptor-mediated glioblastoma cell proliferation and G2/M phase transition. J Biol Chem. 2017;292(21):8933-8947. doi:10.1074/jbc.M116.770420

18. Deng XT, Tang SM, Wu PY, et al. SP/NK-1R promotes gallbladder cancer cell proliferation and migration. J Cell Mol Med. 2019;23 (12):7961-7973. doi:10.1111/jcmm. 14230

19. Munoz M, Rosso M, Covenas R. A new frontier in the treatment of cancer: NK-1 receptor antagonists. Curr Med Chem. 2010;17 (6):504-516. doi:10.2174/092986710790416308

20. Yuan DM, Li Q, Zhang Q, et al. Efficacy and safety of neurokinin-1 receptor antagonists for prevention of chemotherapy-induced nausea and vomiting: systematic review and meta-analysis of randomized controlled trials. Asian Pac J Cancer Prev. 2016;17(4):1661-1675. doi:10.7314/APJCP.2016.17.4.1661

21. Munoz M, Covenas R. Safety of neurokinin-1 receptor antagonists. Expert Opin Drug Saf. 2013;12(5):673-685. doi:10.1517/ 14740338.2013.804059

22. Ge C, Huang H, Huang F, et al. Neurokinin-1 receptor is an effective target for treating leukemia by inducing oxidative stress through mitochondrial calcium overload. Proc Natl Acad Sci U S A. 2019;116(39):19635-19645. doi:10.1073/pnas.190899 8116

23. Kepp O, Galluzzi L, Lipinski M, Yuan J, Kroemer G. Cell death assays for drug discovery. Nat Rev Drug Discov. 2011;10 (3):221-237. doi:10.1038/nrd3373

24. Degterev A, Maki JL, Yuan J. Activity and specificity of necrostatin-1, small-molecule inhibitor of RIP1 kinase. Cell Death Differ. 2013;20(2):366. doi:10.1038/cdd.2012.133

25. Van Noorden CJ. The history of Z-VAD-FMK, a tool for understanding the significance of caspase inhibition. Acta Histochem. 2001;103 (3):241-251. doi:10.1078/0065-1281-00601 
26. Dohner H, Estey EH, Amadori S, et al. Diagnosis and management of acute myeloid leukemia in adults: recommendations from an international expert panel, on behalf of the European LeukemiaNet. Blood. 2010;115(3):453-474.

27. Weick JK, Kopecky KJ, Appelbaum FR, et al. A randomized investigation of high-dose versus standard-dose cytosine arabinoside with daunorubicin in patients with previously untreated acute myeloid leukemia: a Southwest Oncology Group study. Blood. 1996;88 (8):2841-2851. doi:10.1182/blood.V88.8.2841.bloodjournal8882841

28. Bishop JF, Matthews JP, Young GA, et al. A randomized study of high-dose cytarabine in induction in acute myeloid leukemia. Blood. 1996;87(5):1710-1717. doi:10.1182/blood.V87.5.1710. 1710

29. Jensen DD, Lieu T, Halls ML, et al. Neurokinin 1 receptor signaling in endosomes mediates sustained nociception and is a viable therapeutic target for prolonged pain relief. Sci Transl Med. 2017;9(392): eaal3447.
30. Ruiz AL, Martín MVS Neurokinin-1 receptor antagonist composition for treatment of diseases and conditions of the respiratory tract. United States patent US 15/328,825. 2017.

31. Fernandez A, Ordonez R, Reiter RJ, Gonzalez-Gallego J, Mauriz JL. Melatonin and endoplasmic reticulum stress: relation to autophagy and apoptosis. J Pineal Res. 2015;59(3):292-307. doi:10.1111/jpi.12264

32. Corazzari M, Gagliardi M, Fimia GM, Piacentini M. Endoplasmic reticulum stress, unfolded protein response, and cancer cell fate. Front Oncol. 2017;7:78. doi:10.3389/fonc.2017.00078

33. Cai Y, Arikkath J, Yang L, Guo ML, Periyasamy P, Buch S. Interplay of endoplasmic reticulum stress and autophagy in neurodegenerative disorders. Autophagy. 2016;12(2):225-244. doi:10.1080/15548627.2015. 1121360

34. Nakka VP, Prakash-Babu P, Vemuganti R. Crosstalk between endoplasmic reticulum stress, oxidative stress, and autophagy: potential therapeutic targets for acute CNS Injuries. Mol Neurobiol. 2016;53 (1):532-544. doi:10.1007/s12035-014-9029-6

\section{Publish your work in this journal}

Drug Design, Development and Therapy is an international, peerreviewed open-access journal that spans the spectrum of drug design and development through to clinical applications. Clinical outcomes, patient safety, and programs for the development and effective, safe, and sustained use of medicines are a feature of the journal, which has also been accepted for indexing on PubMed Central. The manuscrip management system is completely online and includes a very quick and fair peer-review system, which is all easy to use. Visit http://www. dovepress.com/testimonials.php to read real quotes from published authors. 